\title{
Efectos sobre la flora y vegetación del evento de precipitación extremo de agosto 2015 en Alto Patache, Desierto de Atacama, Chile'
}

\author{
Patricio Pliscoff ${ }^{2}$, Nicolás Zanetta ${ }^{3}$, Josefina Hepp ${ }^{4}$ \\ y Javiera Machuca ${ }^{5}$
}

\begin{abstract}
RESUMEN
Durante el evento extremo "El Niño", ocurrido en agosto de 2015 en la costa de la región de Tarapacá, Desierto de Atacama, se registraron precipitaciones de $50 \mathrm{~mm}$ en el Oasis de Niebla de Alto Patache (promedio anual de $1 \mathrm{~mm}$ ), activando la germinación de muchas de las especies presentes en el lugar. Tras el evento antes mencionado, se demarcaron transectos y se registró la presencia y dominancia de las especies de flora; se compararon los datos florísticos con los registros históricos del sitio y con la distribución de la niebla, medida entre el 2001 y 2002. Como resultado, se identificaron 42 especies, un $78 \%$ del total de flora conocida para el área, con un registro nuevo (Tiquilia sp.). El análisis de las especies, permitió identificar tres unidades de vegetación y se observó una mayor diversidad de especies en la zona con menor altitud del farellon costero.
\end{abstract}

Palabras claves: Desierto de Atacama, Alto Patache, flora, vegetación, neblina.

\begin{abstract}
During the extreme precipitation event related with "El Niño", which occurred in August 2015 on the coast of the Tarapaca region, Atacama Desert, rainfall of $50 \mathrm{~mm}$ was recorded at the Alto Patache Fog Oasis (annual average of $1 \mathrm{~mm}$ ), activating germination of many species present on the site. After the event, transects were demarcated and the presence and dominance of vegetation was recorded. The floristic data identified was compared with historical records and related with the distribution of fog, measured between the years 2001 and 2002. Results show 42 species identified, 78\% of all known plants in the area, with one new record (Tiquilia sp.). The floristic analysis allowed the identification of three vegetation units, and a greater diversity of species was observed in lower areas of the coastal mountain chain.
\end{abstract}

Key words: Atacama Desert, Alto Patache, flora, vegetation, fog.

Artículo recibido el 24 de febrero de 2017, aceptado el 18 de mayo de 2017 y corregido el 14 de septiembre de 2017.

Centro UC Desierto de Atacama / Instituto de Geografía y Departamento de Ecología, Pontificia Universidad Católica de Chile (Chile) E-mail: pliscoff@uc.cl

3 Centro UC Desierto de Atacama / Instituto de Geografía, Pontificia Universidad Católica de Chile (Chile).

E-mail:nczanett@uc.cl

4. Centro UC Desierto de Atacama / Departamento de Ciencias Vegetales, Pontificia Universidad Católica de Chile (Chile). E-mail: jnhepp@uc.cl.

5 Centro UC Desierto de Atacama / Instituto de Geografía, Pontificia Universidad Católica de Chile (Chile).

E-mail: jdmachuc@uc.cl 
El Desierto de Atacama es reconocido como uno de los ecosistemas más singulares del planeta por sus condiciones extremas de aridez. En este ambiente se desarrolla una gran diversidad vegetacional, con presencia de distintas formaciones vegetacionales, como Herbazales, Matorrales y Bosques (Luebert \& Pliscoff, 2017) y un alto número de especies de flora endémica, la cual ha sido, en general, muy poco reconocida (Luebert, 2011; Guerrero et al., 2013; Manrique et al., 2014; Larridon et al., 2015). Estas características de los ecosistemas del Desierto de Atacama se pueden explicar por una gran heterogeneidad ambiental, producto de una activa historia paleo ambiental y de evolución del paisaje (Latorre et al., 2015; Latorre et al., 2013).

El clima del Desierto de Atacama está determinado por un conjunto de factores que le confieren particularidad, entre ellos se encuentran la presencia de una capa de inversión térmica originada por el dominio cuasi permanente del anticiclón del Pacifico sureste, el aporte de aguas frías alimentados por la corriente de Humboldt, surgencias costeras originadas por los vientos del oeste y el efecto de barrera longitudinal que ejerce la cordillera de los Andes (Schulz et al., 2012). Todos estos factores definen un clima extremadamente árido, donde la presencia de precipitaciones es casi nula. Esta condición general de aridez cambia ocasionalmente durante el invierno, relacionado al movimiento de los frentes fríos extratropicales, durante los periodos dominados por el ENSO, en su fase "El Niño" (positiva) el movimiento de los frentes señalados anteriormente puede generar eventos de precipitaciones extremas (Bozcurt et al., 2016).

El fenómeno de El Niño es uno de los elementos que caracterizan la evolución histórica de los paisajes de la zona desértica del norte de Chile (Williams et al., 2008). Durante el periodo temporal en que este fenómeno domina el clima en la zona desértica, se presentan eventos extremos de precipitación, que producen fuertes impactos tanto en el espacio construido por el ser humano (Goldstein \& Magilligan, 2011) como en el natural (Gayó et al., 2012a; Gayó et al., 2012b). Además de la influencia directa del fenómeno de El Niño, se ha identificado que el aumento de temperaturas genera con una mayor frecuencia y magnitud, eventos extremos de precipitación (Ummenhofer \& Meehl, 2017; Westra et al., 2014). En el mes de agosto del año 2015, se produjo uno de dichos eventos, registrándose precipitaciones de $50 \mathrm{~mm}$ concentradas en solo un día (alrededor de $20 \mathrm{~mm}$ en una hora), según los registros de la estación meteorológica ubicada en la Estación UC Oasis Niebla Alto Patache.

La zona de Alto Patache se localiza en el Desierto de Atacama, en la costa de la región de Tarapacá (2049' Latitud Sur y $70^{\circ} 10$ Longitud Oeste) y corresponde a un ecosistema que ha sido identificado como de "lomas" (Dillon, 1989; Rundel et al., 1991) o de "Oasis de Niebla" (Muñoz-Schick et al., 2001; Larrain, 2007); este tipo de ecosistemas se desarrolla desde las costas del Perú, hasta la región de Copiapó en Chile (Dillon \& Hoffmann, 1997). Se caracterizan por la presencia de alta diversidad de flora, asociada a condiciones particulares de humedad, siendo mayor el aporte hídrico que en otras zonas de la costa del desierto por la presencia de la Camanchaca (Cereceda et al., 2004; Latorre et al., 2011).

La flora y vegetación de Alto Patache ha sido caracterizada por diversos estudios (Muñoz-Schick et al., 2001; Egaña et al., 2004; Pinto \& Luebert, 2009), los que han dado cuenta de las especies de flora presentes y de los distintos tipos de vegetación que se pueden encontrar a lo largo del gradiente altitudinal presente. A partir de esta información, más los datos de presencia de flora levantadas en terreno durante campañas en el año 2015, se analizó el efecto del evento extremo 
de precipitación ocurrido en agosto de ese año en la diversidad de flora presente en Alto Patache, caracterizando además la vegetación y su relación con la presencia de la niebla en el área.

\section{Metodología}

Se realizaron dos campañas de registro florístico en el Oasis de Niebla de Alto Patache (Figura $N^{\circ}$ 1). La primera campaña se realizó entre los días 19 y 20 de octubre de 2015, en la ladera sur-oeste, en la cual se realizaron seis transectos horizontales con intervalos de elevación de 100 metros, desde los 350 m s.n.m. hasta los 850 m s.n.m. En cada transecto se identificó la presencia y dominancia de cada especie tomando notas de campo, fotos y ejemplares de referencia para la identificación en laboratorio. Posteriormente, el 3 de diciembre de 2015, se realizó la segunda campaña de recolección en la cual se identificaron y registraron especies ubicadas en la parte superior del área de estudio, sin llevar a cabo transectos específicos para el levantamiento.

\section{Unidades de vegetación}

Una vez realizado el levantamiento de especies en el lugar, estas se agruparon en unidades de vegetación, en función de la presencia y dominancia identificada por rangos altitudinales. La agrupación se sustentó además en las características geomorfológicas y fisiográficas del área de

Figura No 1

Ubicación del área de estudio

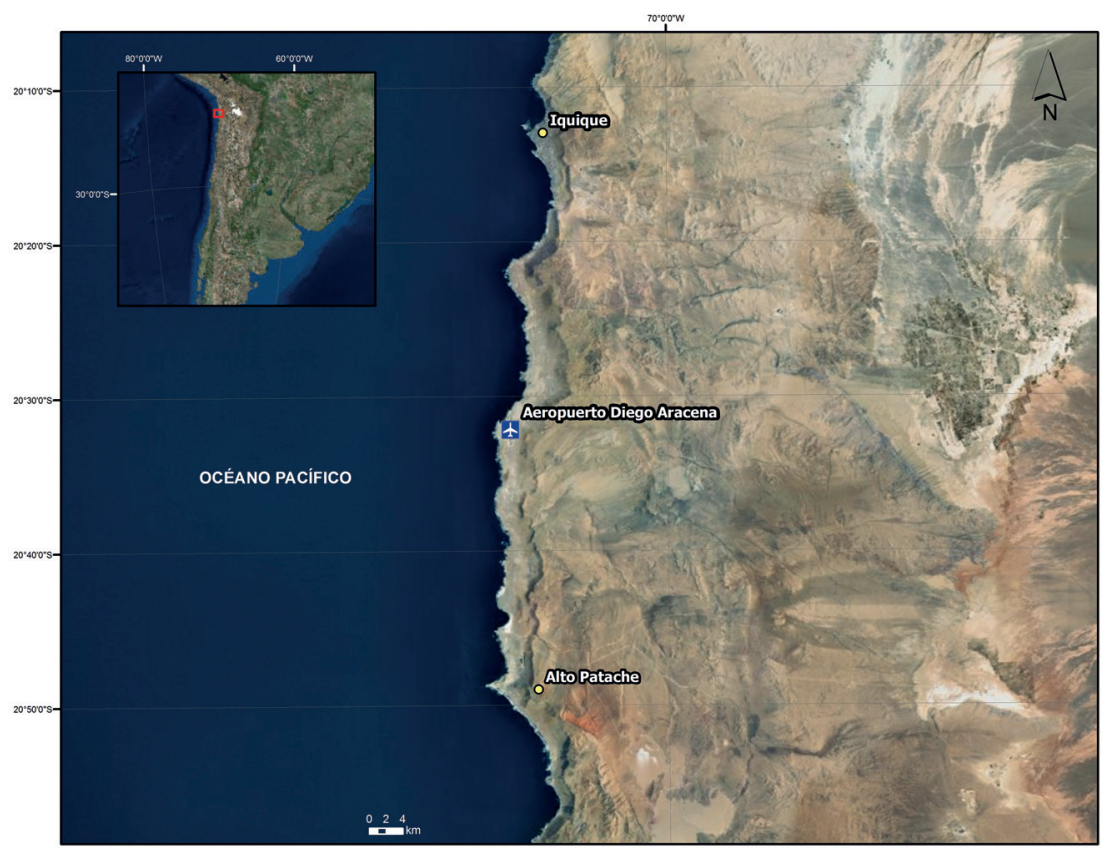

Fuente: Elaboración propia. 
estudio y en las descripciones de vegetación desarrolladas anteriormente tanto para el área como para la zona costera del Desierto de Atacama (Luebert \& Pliscoff, 2006; Gajardo, 1994).

\section{Comparación con la captación de niebla}

Los datos florísticos se analizaron en relación con la altitud en la que fueron identificados y se compararon con la distribución altitudinal de la densidad de la niebla utilizando los datos medidos por los Standard Fog Collectors (SFCs) entre agosto de 2001 y diciembre de 2002, instalados a las mismas altitudes que los transectos realizados para la identificación de especies. Los datos de los SFCs fueron levantados por parte del equipo del Centro UC Desierto de Atacama, información que no había sido publicada hasta ahora. Se consultaron registros históricos y toda la bibliografía disponible sobre la flora vascular del Oasis de Niebla de Alto Patache en particular Muñoz-Schick et al. (2001) y Pinto \& Luebert (2009).

\section{Flora, unidades vegatacionales y comparación de volúmenes de captación de agua}

\section{Flora}

Se identificaron en terreno durante los meses de octubre y diciembre del 2015, 42 especies de flora vascular, correspondiendo a 19 familias y 38 géneros (Cuadro $N^{\circ} 1$ ). La familia más diversa es Solanaceae con nueve especies; el género de especies más diverso identificado es Nolana con cuatro especies (Nolana aplocaryoides, Nolana intonsa, Nolana jaffuelii, Nolana sedifolia). Del total de 42 especies identificadas, 32 corresponden a especies endémicas de Chile. Destaca la presencia de una especie del genero Tiquilia, la cual podría corresponder a T. litoralis, especie que se ha descrito para la costa desértica de Chile, pero que no había sido colectada en esta zona. Las formas de vida más comunes son las plantas con crecimiento arbustivo, seguido de las herbáceas anuales. En relación al total de flora identificado en trabajos anteriores para el área del oasis de Alto Patache, la revisión más completa y exhaustiva corresponde a Pinto \& Luebert (2009), donde se dan cuenta de 54 especies, por lo que el levantamiento realizado en los meses de octubre y diciembre del 2015, alcanzó al 78\% del total de flora ya identificada. En la Figura № 2 se presentan algunas de las especies identificadas en las campañas de terreno.

\section{Cuadro No 1}

Flora identificada en el Oasis de Alto Patache durante las campañas de terreno y la adscripción a las unidades de vegetación

\begin{tabular}{|c|c|c|c|c|}
\hline \multirow[t]{2}{*}{ Familia } & \multirow[t]{2}{*}{ Nombre } & \multicolumn{2}{|c|}{$\begin{array}{l}\text { Campaña de } \\
\text { terreno } 2015\end{array}$} & \multirow{2}{*}{$\begin{array}{l}\text { Unidad de } \\
\text { Vegetación }\end{array}$} \\
\hline & & Octubre & Diciembre & \\
\hline Adiantaceae & Cheilanthes mollis (Kunze) C.Presl & & & 2 \\
\hline Aizoaceae & Tetragonia ovata Phil. & $\mathrm{x}$ & $\mathrm{x}$ & 1,2 \\
\hline Alliaceae & Leucocoryne appendiculata Phil. & $x$ & $x$ & 1,2 \\
\hline
\end{tabular}


Continuación Cuadro No 1

\begin{tabular}{|c|c|c|c|c|}
\hline \multirow[t]{2}{*}{ Familia } & \multirow[t]{2}{*}{ Nombre } & \multicolumn{2}{|c|}{$\begin{array}{l}\text { Campaña de } \\
\text { terreno } 2015\end{array}$} & \multirow{2}{*}{$\begin{array}{l}\text { Unidad de } \\
\text { Vegetación }\end{array}$} \\
\hline & & Octubre & Diciembre & \\
\hline & Nothoscordum sp. & $x$ & $x$ & 1 \\
\hline $\begin{array}{l}\text { Alstroemeria- } \\
\text { ceae }\end{array}$ & Alstroemeria lutea M.Muñoz-Schick & $x$ & & 2 \\
\hline \multirow[t]{3}{*}{ Amaranthaceae } & Atriplex taltalensis I.M.Johnst. & $x$ & & 2 \\
\hline & Chenopodium petiolare Kunth & $x$ & $x$ & 3 \\
\hline & Suaeda foliosa Moq. & $x$ & & 3 \\
\hline Apiaceae & $\begin{array}{l}\text { Cyclospermum laciniatum (DC.) } \\
\text { Constance }\end{array}$ & & & 3 \\
\hline \multirow[t]{6}{*}{ Asteraceae } & Amblyopappus pusillus Hook. et Arn. & $x$ & $x$ & 3 \\
\hline & $\begin{array}{l}\text { Ophryosporus floribundus (DC.) R.M. King } \\
\text { et H. Rob. }\end{array}$ & $x$ & & 2 \\
\hline & Perityle emoryi Torr. & & & 1 \\
\hline & Polyachyrus annuus I.M.Johnst. & $x$ & $x$ & 1,2 \\
\hline & Polyachyrus sphaerocephalus D.Don & & & 1 \\
\hline & Sonchus tenerrimus L. & & & 1 \\
\hline Bignoniaceae & Argylia radiata (L.) D.Don & $x$ & & 1 \\
\hline \multirow[t]{2}{*}{ Boraginaceae } & Cryptantha filiformis (Phil.) Reiche & $x$ & & 1,3 \\
\hline & Tiquilia sp. & & $x$ & 3 \\
\hline Brassicaceae & Cleome chilensis DC. & & & 1 \\
\hline \multirow[t]{3}{*}{ Cactaceae } & $\begin{array}{l}\text { Cumulopuntia sphaerica (C.F.Först.) } \\
\text { E.F.Anderson }\end{array}$ & $x$ & $x$ & 1,2 \\
\hline & Eriosyce caligophila R.Pinto & $x$ & & 2 \\
\hline & $\begin{array}{l}\text { Eulychnia iquiquensis (K.Schum.) Britton } \\
\text { et Rose }\end{array}$ & $x$ & $x$ & 1,2 \\
\hline \multirow[t]{2}{*}{ Caryophyllaceae } & $\begin{array}{l}\text { Spergularia aff. arbuscula (Gay) } \\
\text { I.M.Johnst. }\end{array}$ & & & 3 \\
\hline & Spergularia stenocarpa (Phil.) I.M.Johnst. & & & 3 \\
\hline Ephedraceae & Ephedra breana Phil. & $x$ & & 2,3 \\
\hline \multirow[t]{3}{*}{ Fabaceae } & Astragalus sp. & & & 1 \\
\hline & Astragalus triflorus (DC.) A.Gray & $x$ & $x$ & 1 \\
\hline & Hoffmannseggia prostrata Lag. ex DC. & $x$ & $x$ & 1,3 \\
\hline Frankeniaceae & Frankenia chilensis C.Presl & $x$ & $x$ & 2,3 \\
\hline Hyacinthaceae & Oziroë biflora (Ruiz et Pav.) Speta & $x$ & $x$ & 1,2 \\
\hline Iridaceae & Olsynium scirpoideum (Poepp.) Goldblatt & $x$ & $x$ & 1 \\
\hline Loasaceae & Loasa nitida Desr. & $x$ & & 1 \\
\hline Malesherbiaceae & Malesherbia multiflora Ricardi & $x$ & $x$ & 3 \\
\hline Malvaceae & Cristaria integerrima Phil. & $x$ & $x$ & 1 \\
\hline
\end{tabular}


Continuación Cuadro No 1

\begin{tabular}{|c|c|c|c|c|}
\hline \multirow[t]{2}{*}{ Familia } & \multirow[t]{2}{*}{ Nombre } & \multicolumn{2}{|c|}{$\begin{array}{l}\text { Campaña de } \\
\text { terreno } 2015\end{array}$} & \multirow{2}{*}{$\begin{array}{l}\text { Unidad de } \\
\text { Vegetación }\end{array}$} \\
\hline & & Octubre & Diciembre & \\
\hline & Cristaria molinae Gay & $x$ & $x$ & 3 \\
\hline & Cristaria pinnata Phil. & $x$ & $\mathrm{x}$ & 1 \\
\hline Nyctaginaceae & Mirabilis elegans (Choisy) Heimerl & $x$ & $\mathrm{x}$ & 1 \\
\hline Oxalidaceae & Oxalis bulbocastanum Phil. & $x$ & $x$ & 1,2 \\
\hline Poaceae & Jarava vaginata (Phil.) F.Rojas & $x$ & & 2,3 \\
\hline Polemoniaceae & Bryantiella glutinosa (Phil.) J.M.Porter & & & 1 \\
\hline \multirow[t]{3}{*}{ Portulacaceae } & Calandrinia litoralis Phil. & $x$ & $x$ & 1,3 \\
\hline & $\begin{array}{l}\text { Cistanthe amaranthoides (Phil.) Carolin ex } \\
\text { Hershk. }\end{array}$ & $\mathrm{x}$ & $\mathrm{x}$ & 3 \\
\hline & $\begin{array}{l}\text { Cistanthe grandiflora (Lindl.) Carolin ex } \\
\text { Hershk. }\end{array}$ & $\mathrm{x}$ & $x$ & 3 \\
\hline \multirow[t]{9}{*}{ Solanaceae } & Quinchamalium chilense Molina & $x$ & & 2,3 \\
\hline & Lycium cf. deserti Phil. & $\mathrm{x}$ & & 3 \\
\hline & $\begin{array}{l}\text { Nolana aplocaryoides (Gaudich.) } \\
\text { I.M.Johnst. }\end{array}$ & $\mathrm{x}$ & $\mathrm{x}$ & 3 \\
\hline & Nolana intonsa I.M.Johnst. & $x$ & $x$ & 2,3 \\
\hline & Nolana jaffuelii I.M.Johnst. & $x$ & $x$ & $1,2,3$ \\
\hline & Nolana sedifolia Poepp. & $x$ & $x$ & 2 \\
\hline & Solanum brachyantherum Phil. & $x$ & $x$ & 2,3 \\
\hline & Solanum cfr. Montanum L. & & & 3 \\
\hline & Solanum chilense (Dunal) Reiche & & & 3 \\
\hline Tecophilaeaceae & Zephyra elegans D.Don & $x$ & $x$ & 1,2 \\
\hline Urticaceae & Parietaria debilis G.Forst. & & $x$ & 1 \\
\hline Verbenaceae & $\begin{array}{l}\text { Glandularia atacamensis (Reiche) } \\
\text { J.M.Watson et A.E.Hoffm. }\end{array}$ & & & 1,2 \\
\hline
\end{tabular}

Fuente: Elaboración propia.

\section{Unidades de vegetación}

A partir de los transectos y la identificación de especies realizadas, se caracterizaron tres unidades de vegetación, considerando la presencia y dominancia de especies, las características de sustrato y la geomorfología presente. Estas tres unidades se presentan en un gradiente altitudinal desde el farellón costero hasta la zona más alta de la cordillera de la costa (Figura No 3). Las Unidades de vegetación descritas son las siguientes:

- Unidad de Vegetación No 1: Herbazal efímero de Nolana jaffueli, ubicado en la zona del farellón costero bajo los 600 m s.n.m. Esta unidad se caracteriza por estar compuesta ma- 
Figura No 2

Algunas especies presentes en el oasis de niebla de Alto Patache. A. Zephyra elegans, B. Hoffmannseggia prostrata, C. Tetragonia ovata, D. Alstroemeria lutea, E. Nolana jaffuelii y Cristaria molinae, F. Frankenia chilensis, G. Solanum brachyantherum, H. Tiquilia sp, I. Oxalis bulbocastanum, J. Calandrinia litoralis, K. Leucocoryne appendiculata, L. Cryptantha filiformis.
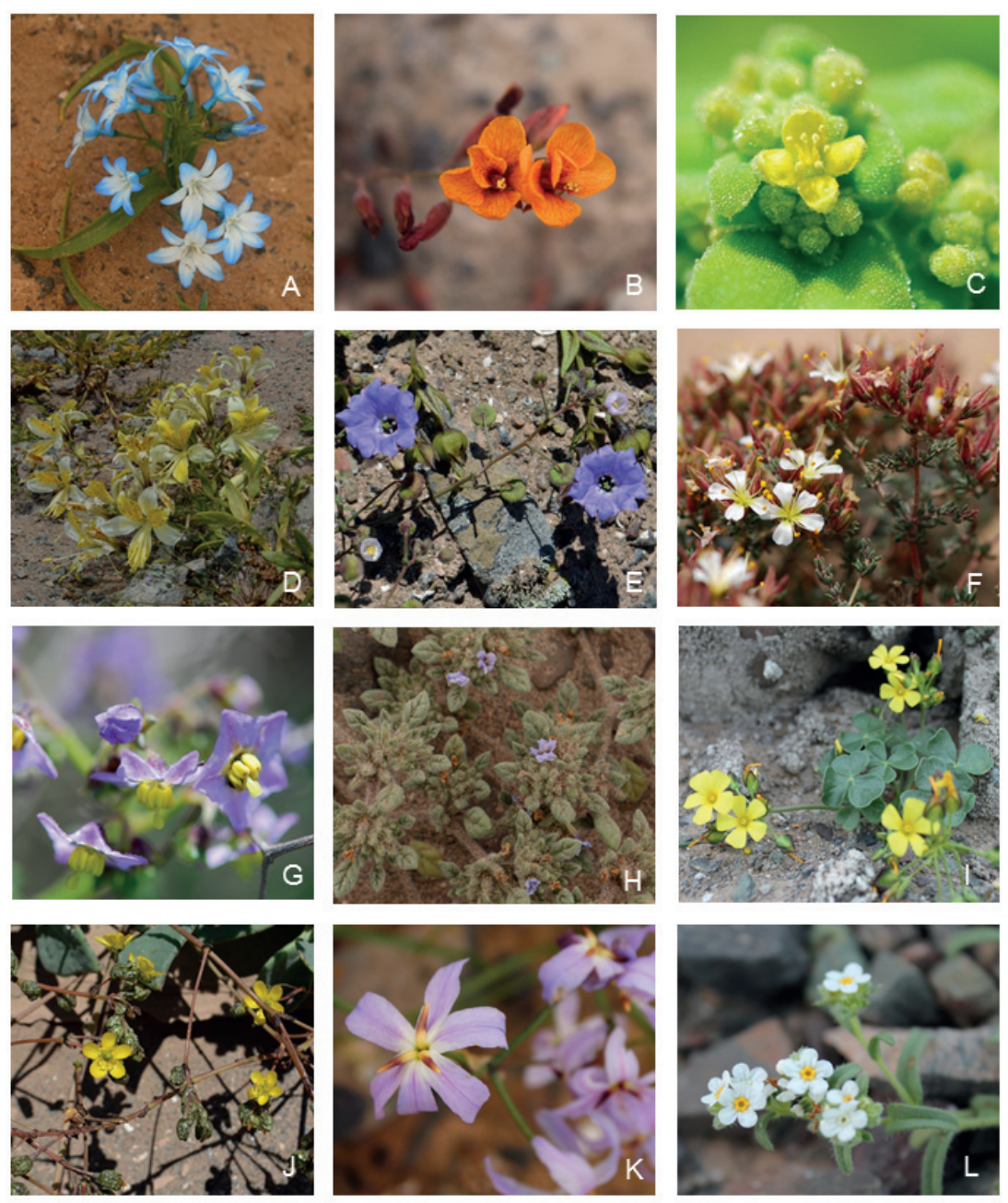

Fuente: Elaboración propia.

yoritariamente por especies herbáceas anuales, dominando la especie Nolana jaffueli. Esta unidad presenta la mayor diversidad de especies identificada para el área (28), y corresponde a la unidad que depende más directamente del aporte de precipitación y que, por lo tanto, posee una mayor variación interanual en términos de riqueza específica; durante periodos secos, esta unidad presenta una cobertura y diversidad muy baja. Otras especies relevantes que caracterizan esta unidad; Cleome chilensis, Argylia radiata, Cistanthe intergerrima y Eulychnia chilensis. 
- Unidad de Vegetación № 2: Matorral rupícola de Nolana sedifolia, esta unidad se presenta entre los 800 y los 600 m s.n.m. en zonas altas del farellón costero, asociada a un sustrato rocoso, que permite una mayor variabilidad de ambientes bajo distintas condiciones microclimáticas. Esta unidad en algunas zonas se mezcla con la primera, presentado también una alta diversidad a nivel especifico. Es aquí donde se identifican algunas de las especies más escasas y particulares del área, como Alstroemeria lutea, la cactácea Eryosice caligophila y el helecho Cheilanthes mollis. Otras especies relevantes que caracterizan esta unidad; Atriplex taltalensis, Ephedra breana, Oxalis bulbocastanum, Frankenia chilensis.

- Unidad de Vegetación No 3: Matorral muy abierto de Nolana aplocaryoides, corresponde a las zonas más altas de la cordillera de la Costa, al área que se proyecta hacia el interior del desierto sobre los $800 \mathrm{~m}$ s.n.m. En términos de relieve, esta área posee el menor gradiente altitudinal, siendo una unidad homogénea con un relieve plano. En algunas zonas de esta unidad se identifican aguadas inactivas, que entregan condiciones de microrelieve propicio para el establecimiento de algunas especies en especial durante los eventos de lluvias, que también se identifican en otras unidades. Otras especies relevantes que caracterizan esta unidad; Malesherbia multiflora, Cistanthe amaranthoides, Cristaria molinae, Solanum chilense.

En relación a las descripciones anteriores de vegetación para el área, se puede establecer una correspondencia entre la Unidad de vegetación No 1, Herbazal efímero de Nolana Jaffueli y el piso de vegetación de Matorral desértico tropical costero de Nolana adansonii y N. lycioides, descrito por Luebert \& Pliscoff (2006). Así como también, elementos florísticos descritos para la Unidad de Vegetación $\mathrm{N}^{\circ} 2$ y $\mathrm{N}^{\circ} 3$, se encuentran en las descripciones del piso de vegetación de Matorral

Figura No 3

Unidades de vegetación del oasis de Alto Patache

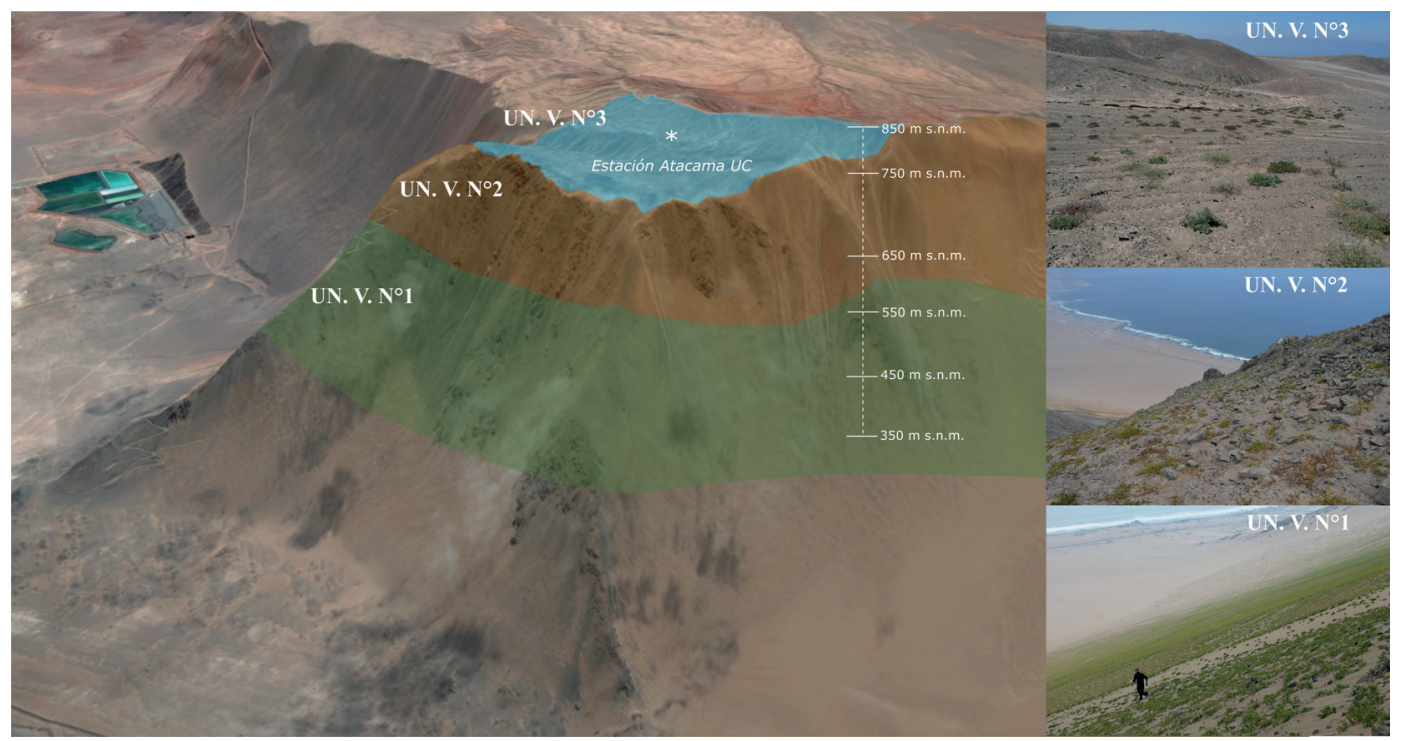

Fuente: Elaboración propia. 
desértico tropical costero de Ephedra breana y Eulychnia iquiquensis; sin embargo, en este caso no se puede establecer una correspondencia tan directa por las diferencias de escala entre los dos estudios, ya que los pisos de vegetación corresponden a una clasificación de vegetación zonal escala 1:100.000, diferente a lo analizado en este trabajo.

\section{Comparación con la captación de neblina}

Los volúmenes de captación de agua de niebla en el acantilado costero del Oasis de Niebla de Alto Patache presentan un marcado ciclo anual (Figura $N^{\circ} 4$ ). Estos alcanzan valores máximos en los meses de invierno tardío y primavera temprana (julio, agosto y septiembre - JAS) con un promedio de $13.7 \mathrm{~L} \mathrm{~m}^{-2} \mathrm{~d}^{-1}$ a los $850 \mathrm{~m}$ s.n.m. Por otro lado, durante los meses de verano (enero, febrero y marzo - EFM), se presentan los volúmenes de agua de niebla más bajos del año con promedios cercanos a $2 \mathrm{~L} \mathrm{~m}^{-2} \mathrm{~d}^{-1}$ a esta misma altitud analizada (Pinto et al., 2001; Cereceda et al., 2004).

El ciclo anual de la captación de agua de niebla es similar en todo el acantilado, sin embargo, los volúmenes captados son muy distintos. La vertiente sur-oeste del acantilado costero en el área de estudio presenta una marcada variabilidad altitudinal en los volúmenes de agua de niebla, concentrando los valores más altos a los 850 y $750 \mathrm{~m}$ s.n.m. con $2.1 \mathrm{~L} \mathrm{~m}^{-2} \mathrm{~d}^{-1}$ y $1.2 \mathrm{~L} \mathrm{~m}^{-2} \mathrm{~d}^{-1}$ como promedio anual, respectivamente. Los volúmenes de agua captados disminuyen drásticamente a medida que disminuye la altitud en la que se registran, con valores de $0,17 \mathrm{~L} \mathrm{~m}^{-2} \mathrm{~d}^{-1}$ a los $650 \mathrm{~m}$ s.n.m. y $0,079 \mathrm{~L} \mathrm{~m}^{-2} \mathrm{~d}^{-1}$ a los $550 \mathrm{~m}$ s.n.m. Bajo este umbral, los registros son prácticamente nulos durante todo el año, con volúmenes mínimos de agua que son captados solo durante los meses de invierno (junio a septiembre).

Figura $\mathrm{N}^{\circ} 4$

Estructura de la niebla en el acantilado costero de la región de Tarapacá

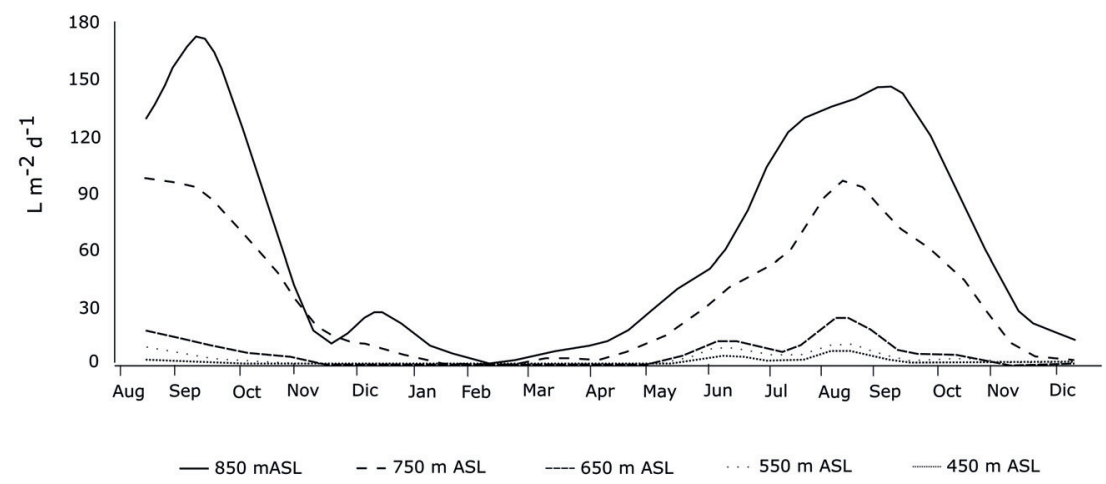

Fuente: Elaboración propia.

Los datos de volúmenes de agua de niebla captada no presentan relación con el número de especies registradas por unidad Vegetacional (Cuadro $N^{\circ} 2$ ) en este evento, lo que no quiere decir que no sea un factor de aporte hídrico para ellas. El número de especies por unidad Vegetacional podría tener una relación más directa con otras condiciones del territorio como las características del sustrato donde se encuentran, la distribución de la niebla a través del micro- 
relieve, e incluso el impacto de las precipitaciones sobre el terreno pudiendo desplazar semillas hacia los sectores más bajos del acantilado.

Los volúmenes de agua de niebla captados en promedio en las Unidades de Vegetación y el número de especies identificadas en estas, se presentan en el Cuadro № 2.

Cuadro No 2

Volumen de captación de agua de niebla y número de especies por Unidad Vegetacional (UN.V.)

\begin{tabular}{|c|r|r|r|}
\hline UN.V. & Rango altitud (m s.n.m.) & Niebla $\left(\mathrm{L} \mathrm{m}^{-2} \mathrm{~d}^{-1}\right)$ & $\mathrm{N}^{\circ}$ Especies \\
\hline 1 & $(-) 600$ & 0,06 & 28 \\
\hline 2 & $800-600$ & 0,70 & 22 \\
\hline 3 & $(+) 800$ & 2,13 & 25 \\
\hline
\end{tabular}

Fuente: Elaboración propia.

\section{Conclusión}

Este trabajo permitió evaluar la flora y vegetación presente en el área del oasis de Alto Patache, después del evento extremo de precipitación del mes de agosto del 2015 y relacionarlo con la captación de neblina en el sector. En relación a la flora identificada destaca el hecho de que se logró relevar casi el $80 \%$ de las especies descritas por el conjunto de trabajos anteriores, los cuales se basaron en relevamientos mucho más intensivos tanto en términos temporales como de cobertura geográfica.

Esto da cuenta de la relevancia del aporte de las precipitaciones para la vegetación en el desierto, en particular para las herbáceas anuales y perennes, ya que estos raros eventos representan una oportunidad de reproducción, dispersión y establecimiento para estas especies, además de reponer el banco de semillas del suelo (Gutiérrez et al., 2000; Schulz et al., 2011). Una cantidad de lluvia como la de agosto de 2015, que probablemente sobrepasó el umbral de requerimiento hídrico de la mayoría de las especies presentes y gatilló su germinación (Gutiérrez et al., 2000), también pudo haber desplazado semillas hacia los sectores más bajos del oasis, junto con los aluviones que se produjeron a distintas escalas.

El aporte del agua de niebla, en tanto, también sería significativo, pero estaría más relacionado con la mantención de la vegetación, en particular de especies arbustivas que permanecen sobre la superficie, ya que la niebla las protege de la radiación solar directa, reduce las tasas de evapotranspiración al mantener temperaturas bajas y alta humedad atmosférica, y también provee de agua a ciertas taxa adaptadas a la captación de agua de niebla (Schulz et al., 2011). El sustrato rocoso que se encuentra en la Unidad No 2, y en algunos puntos específicos de las otras unidades, actuaría interceptando el agua de niebla y permitiendo el crecimiento de gran cantidad de especies a su alrededor. 


\section{Referencias bibliográficas}

BOZKURT, D.; RONDANELLI, R.; GARREAUD, R. \& ARRIAGADA, A. Impact of Warmer Eastern Tropical Pacific SST on the March 2015 Atacama Floods. Monthly Weather Review, 2016, Vol. 144, No 11, p. 4441-4460.

CERECEDA, P.; PINTO, R.; LARRAIN, H.; OSSES, P. \& FARÍAS, M. Geographical Description of Three Fog Ecosystems in the Atacama Coastal Desert of Chile. Third International Conference on Fog, Fog Collection and Dew. Ciudad Del Cabo, Sudáfrica, 2004.

DILLON, M. Origins and diversity of the lomas formations in the Atacama and Peruvian Deserts of western South America. American Journal of Botany, 1989, Vol. 76, No 6, p. 950.

DILLON, M. \& HOFFMANN, A. E. Lomas formations of the Atacama Desert, northern Chile. In: DAVIS, S.D.; HEYWOOD, V.H.; HERRERA-MCBRYDE, O.; VILLA-LOBOS, J. \& HAMILTON, A.C. (editors). Centres of Plant Diversity, A Guide and Strategy for their Conservation. Oxford: WWF, Information Press, 1997, p. 528-535.

EGAÑA, I.; CERECEDA, P.; PINTO, R.; LARRAÍN, H.; OSSES, P. y FARÍAS, M. Estudio biogeográfico de la comunidad arbustiva del farellón costero de Punta Patache, Iquique, Chile. Revista de Geografía Norte Grande, 2004, No 31, p. 99-113.

GAJARDO, R. La vegetación natural de Chile. Clasificación y distribución geográfica. Santiago de Chile: Editorial Universitaria, 1994.

GAYÓ, E.M.; LATORRE, C.; SANTORO, C.M.; MALDONADO, A. \& DE POL-HOLZ, R. Hydroclimate variability in the low-elevation Atacama Desert over the last 2500 yr. Climate of the Past, 2012, No 8, p. 287-306.

GAYÓ, E.M.; LATORRE, C.; JORDAN, T.E.; NESTER, P.L.; ESTAY, S.E.; OJEDA, K.F. \& SANTORO, C.M. Late Quaternary hydrological and ecological change in the hyperarid core of the northern Atacama Desert ( $\left.21^{\circ} \mathrm{S}\right)$. Earth-Science Reviews, 2012, No 113, p. 120-140.

GOLDSTEIN, P.S. \& MAGILLIGAN, F.J. Hazard, risk and agrarian adaptations in a hyperarid watershed: El Niño floods, streambank erosion, and the cultural bounds of vulnerability in the Andean Middle Horizon. Catena, 2011, Vol. 85, No 2, p. 155-167.

GUERRERO, P.C.; ROSAS, M.; ARROYO, M.T.K. \& WIENS, J.J. Evolutionary lag times and recent origin of the biota of an ancient desert (Atacama-Sechura). Proceedings of the National Academy of Sciences, 2013, Vol. 110, No 28, p. 11469-11474.

GUTIÉRREZ, J.R.; ARANCIO, G. \& JAKSIC, F. Variation in vegetation and seed bank in a Chilean semi-arid community affected by ENSO 1997. Journal of Vegetation Science, 2000, N 11, p. 641-648.

LARRAIN, B. Relaciones florísticas entre oasis de neblina del desierto costero del norte de Chile. Santiago de Chile: Memoria de Titulo, Facultad de Ciencias Agronómicas, Universidad de Chile, 2007. 
LARRIDON, I.; WALTER, H. E.; GUERRERO, P.C.; DUARTE, M.; CISTERNAS, M.A.; HERNÁNDEZ, C.P.; BAUTERS, K.; ASSELMAN, P.; GOETGHEBEUR, P. \& SAMAIN, M.S. An integrative approach to understanding the evolution and diversity of Copiapoa (Cactaceae), a threatened endemic Chilean genus from the Atacama Desert. American Journal of Botany, 2015, Vol. 102, No 9, p. 1506-1520.

LATORRE, C.; GONZÁLEZ, A.L.; QUADE, J.; FARIÑA, J.M.; PINTO, R. \& MARQUET, P.A. Establishment and formation of fog-dependent Tillandsia landbeckii dunes in the Atacama Desert: Evidence from radiocarbon and stable isotopes. Journal of Geophysical Research, 2011, № 116, p. G03033.

LATORRE, C.; SANTORO, C.; UGALDE, P.; GAYO, E.; OSORIO, D.; SALAS-EGAÑA, C.; DE POL-HOLZ, R.; JOLY, D. \& RECH, J. Late Pleistocene human occupation of the hyperarid core in the Atacama Desert, northern Chile. Quaternary Science Reviews, 2013, № 77, p. 19-30.

LATORRE, C.; MUJICA, I.; MALDONADO, A.; GONZALEZ-SILVESTRE, L; PINTO, R.; DE POL-HOLZ, R. \& SANTORO, C. Late Quaternary climate change, relict populations and present-day refugia in the northern Atacama Desert: a case study from Quebrada La Higuera (18 degrees S). Journal Of Biogeography, 2015, Vol. 42, No 1, p. 76-88.

LUEBERT, F. Hacia una fitogeografía histórica del Desierto de Atacama. Revista Geografía Norte Grande, 2011, № 50, p. 105-133.

LUEBERT, F. y PLISCOFF, P. Sinopsis bioclimática y vegetacional de Chile. Santiago de Chile: Editorial Universitaria, 2016.

LUEBERT, F. y PLISCOFF, P. Sinopsis bioclimática y vegetacional de Chile. Santiago de Chile: Editorial Universitaria, Segunda Edición, 2017.

MANRIQUE, R.; RICOTTA, C.; FERRARI, C. \& PEZZI, G. Latitudinal pattern in plant composition along the Peruvian and Chilean fog oases. Plant Biosystems, 2014, Vol. 148, No 5, p. 1002-1008.

MUÑOZ-SCHICK, M.; PINTO, R.; MESA, A. y MOREIRA-MUÑOZ, A. Oasis de neblina en los cerros costeros del sur de lquique, región de Tarapacá, Chile, durante el evento El Niño 1997-1998. Revista Chilena de Historia Natural, 2001, No 74, p. 389-405.

PINTO, R.; LARRAÍN, H.; CERECEDA, P.; LÁZARO, P.; OSSES, P. \& SCHEMENAUER, R.S. Monitoring fog-vegetation communities at a fog-site in Alto Patache, South of lquique, Northern Chile, during "El Niño" and "La Niña" events (1997-2000). In: SCHEMENAUER, R.S. \& BRIDGMAN, H. (editors). Proceedings Second Conference on Fog and Fog Collection. St. John's, Canadá, 2001, p. 293-296.

PINTO, R. y LUEBERT, F. Datos sobre la flora vascular del desierto costero de Arica y Tarapacá, Chile, y sus relaciones fitogeográficas con el sur de Perú. Gayana Botánica, 2009, Vol. 66, № 1, p. 28-49.

RUNDEL, P.; DILLON, M.; PALMA, B.; MOONEY, H.; GULMON, S. \& EHLERINGER, J.R. The phytogeography and ecology of the coastal Atacama and Peruvian deserts. Aliso, 1991, Vol. 13, No 1, p. 1-49. 
SCHULZ, N.; ACEITUNO, P. \& RICHTER, M. Phytogeographic divisions, climate change and plant dieback along the coastal desert of Northern Chile. Erdkunde, 2011, №65, p. 169-187.

SCHULZ, N.; BOISIER, J.P. \& ACEITUNO, P. Climate change along the arid coast of northern Chile. International Journal of Climatology, 2012, Vol. 32, No 12, p. 1803-1814.

UMMENHOFER, C.C. \& MEEHL, G.A. Extreme weather and climate events with ecological relevance: a review. Philosophical Transactions of the Royal Society of London B: Biological Sciences, 2017, Vol. 372, No 1723, p. 20160135.

WESTRA, S.; FOWLER, H.J.; EVANS, J.P.; ALEXANDER, L.V.; BERG, P.; JOHNSON, F.; KENDON, E.J.; LENDERINK, G. \& ROBERTS NM. Future changes to the intensity and frequency of short-duration extreme rainfall. Reviews of Geophysics, 2014, Vol. 201, No 52, p. 522-555.

WILLIAMS, A.; SANTORO, C.; SMITH, M. \& LATORRE, C. The impact of Enso in the Atacama Desert and Australian arid zone: Exploratory time series analysis of archaeological records. Chungará, 2008, No 40, p. 245-259. 
